\title{
Shock tóxico por Streptococcus agalactiae, a propósito de un caso
}

\author{
Toxic shock from Streptococcus agalactiae, about a case
}

Noel J. Díaz-Robles ${ }^{1 *}$, Mariano A. Chávez-Andino ${ }^{1}$, Cecilia E. Rivera-Díaz y y Óscar A. Santos-Urbina ${ }^{1}$

${ }^{1}$ Servicio de Medicina Crítica de Adultos; ${ }^{2}$ Servicio de Infectología de Adultos. Hospital Médico Quirúrgico del Instituto Salvadoreño del Seguro Social, San Salvador, El Salvador

\section{Introducción}

Streptococcus agalactiae, también denominado estreptococo del grupo B (SGB), se ha asociado con una carga cada vez mayor de enfermedad invasiva. El riesgo de infección por SGB se consideró, durante años, más frecuente en los recién nacidos.

Los estudios muestran que las tasas poblacionales han aumentado significativamente en todas las edades en las últimas décadas, pero los incrementos más pronunciados han sido en pacientes mayores de 15 años, cuyas tasas pasaron de 0.92 a 2.39 por 100,000 habitantes, una media del $6 \%$ anual ${ }^{1}$.

Además, para 2010, los adultos contribuyeron con el $66 \%$ de todos los informes de SGB invasivos, en comparación con el $51 \%$ en $1991^{1}$. También se han observado aumentos en la enfermedad de adultos en los Estados Unidos de América y Noruega ${ }^{1}$.

El $17 \%$ al $43 \%$ de las bacteriemias por SGB son polimicrobianas, a menudo en combinación con estafilococos ${ }^{2-5}$.

Presentamos el caso de un paciente con infección subcutánea necrotizante, inestabilidad hemodinámica y falla renal aguda, con un rápido desarrollo de síntomas graves, documentándose posteriormente la presencia de bacteriemia por $S$. agalactiae, que englobó un síndrome de shock tóxico por SGB, con evolución satisfactoria.

\section{Descripción del caso}

Hombre de 86 años, procedente y residente del departamento de La Libertad, pensionado, que consulta con historia de 4 días de presentar edema ascendente de miembros inferiores que se acompañó de coloración violácea, y fiebre cuantificada de $40^{\circ} \mathrm{C}$ de tipo continuo que no cedía con analgésicos, además de escalofríos y diaforesis. Dos días antes de su consulta acusa adinamia e hiporexia. El paciente tiene antecedente de hipertensión arterial manejada con amlodipino $10 \mathrm{mg}$, una tableta cada día desde hace más o menos 15 años.

En la unidad de emergencia se encuentra con presión arterial de 70/40 mmHg, frecuencia cardíaca de 138 latidos/minuto, frecuencia respiratoria de 26 respiraciones/minuto, saturación de oxígeno del 95\%, Glasgow de 15 puntos, celulitis de ambos miembros inferiores y esfacelación acompañada de bullas (Figs. 1 y 2).

Continúa con hipotensión arterial, a pesar de la reanimación hídrica vigorosa, con necesidad de iniciar tratamiento vasopresor (noradrenalina $20 \mu \mathrm{g} / \mathrm{min}$ ). Es manejado de manera empírica con vancomicina y trasladado a la unidad de cuidados intensivos (UCI).

Los exámenes de laboratorio sugerían un proceso infeccioso y el hemograma mostraba leucocitosis, con neutrofilia, proteína $C$ reactiva alta, procalcitonina elevada, creatinina elevada y electrolitos normales (Tabla 1).

\section{Correspondencia:}

*Noel J. Díaz-Robles

E-mail: dr.diazrobles86@gmail.com
Fecha de recepción: 02-09-2021

Fecha de aceptación: 07-12-2021 DOI: 10.24875/REIE.21000097
Disponible en internet: 07-03-2022

Rev Educ Investig Emer. 2022;4(Supl 1):55-58 www.medicinadeemergencias.com

列 bajo la licencia CC BY-NC-ND (http://creativecommons.org/licenses/by-nc-nd/4.0/). 


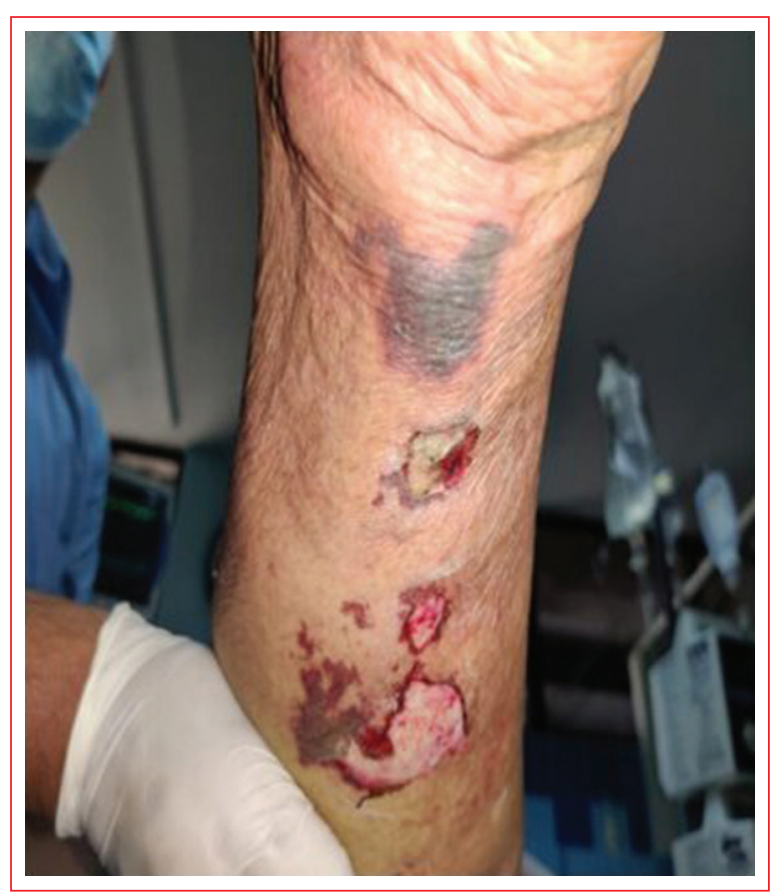

Figura 1. Extremo inferior de pierna izquierda.

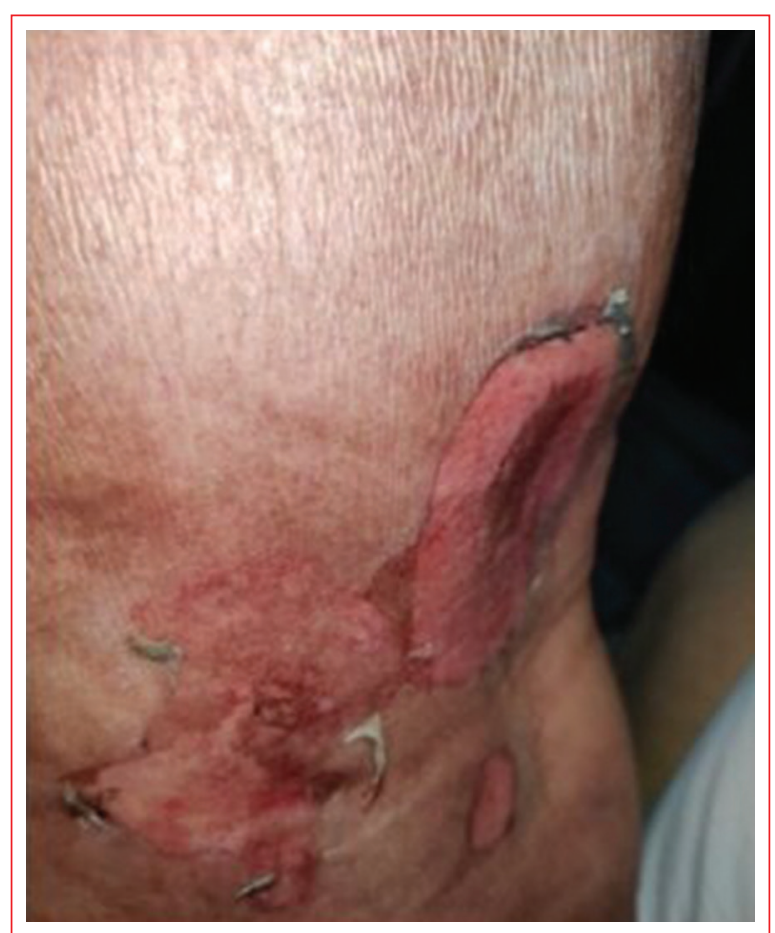

Figura 2. Parte posterior de muslo derecho.

Hacia el segundo día de hospitalización en la UCI presenta deterioro hemodinámico, requiriendo mayores dosis de noradrenalina ( $80 \mu \mathrm{g} / \mathrm{min})$, y reporte preliminar de
Tabla 1. Reporte de los exámenes del caso presentado

\begin{tabular}{|l|c|}
\hline Parámetro & Valor \\
\hline Hemoglobina & $12.1 \mathrm{~g} / \mathrm{dl}$ \\
\hline Leucocitos & $15,700 \mathrm{~mm}^{3}$ \\
\hline Neutrófilos & $93.4 \%$ \\
\hline Linfocitos & $3.0 \%$ \\
\hline Plaquetas & $185,000 \mathrm{UL}$ \\
\hline Proteína C reactiva & $56.2 \mathrm{mg} / \mathrm{dl}$ \\
\hline Glucosa & $130 \mathrm{mg} / \mathrm{dl}$ \\
\hline Creatinina & $2.4 \mathrm{mg} / \mathrm{dl}$ \\
\hline Urea & $146 \mathrm{mg} / \mathrm{dl}$ \\
\hline Sodio & $146 \mathrm{mmol} / \mathrm{l}$ \\
\hline Potasio & $4.7 \mathrm{mmol} / \mathrm{l}$ \\
\hline Procalcitonina & $7.2 \mathrm{ng} / \mathrm{ml}$ \\
\hline Hemocultivos periféricos & Streptococcus agalactiae \\
\hline
\end{tabular}

dos hemocultivos periféricos positivos para cocos grampositivos en cadena. Se descartó insuficiencia vascular de miembros inferiores por ultrasonografía Doppler.

El tercer día se reporta la sensibilidad de los hemocultivos periféricos ya descritos y se ajusta el manejo antibiótico con penicilina $\mathrm{G}$ sódica (4 millones de unidades cada 4 horas) y clindamicina (900 mg cada 8 horas). Paulatinamente se logran el destete del soporte vasopresor y la normalización de la función renal. El destete completo del soporte vasopresor se logró hacia el quinto día de estancia en la UCl. Continúa con antibióticos por 14 días, y dos hemocultivos periféricos de control muestran la resolución del cuadro de bacteremia.

La presunción diagnóstica de síndrome de shock tóxico por $S$. agalactiae se fundamentó en el deterioro hemodinámico, el requerimiento de vasopresor (noradrenalina $80 \mu \mathrm{g} / \mathrm{min}$ ), la falla renal aguda, la celulitis de miembros inferiores y la evidencia de dos hemocultivos periféricos positivos para SGB. El paciente fue dado de alta con manejo ambulatorio de curaciones diarias de los miembros inferiores.

\section{Discusión}

El inicio repentino y expresivo de los síntomas en este caso es notable y se correlaciona con el daño 
potencial que puede traer esta infección. Tales hallazgos son representativos del papel de las toxinas estreptocócicas en este escenario, más que de la agresividad de la propia bacteria.

La erupción se limita a las áreas de drenaje linfático inadecuado, simulando una infección cutánea localizada, como una celulitis. Condiciones como linfedema, insuficiencia vascular, dermatitis crónica o lesión cutánea inducida por radiación son factores predisponentes a las infecciones de la piel y los tejidos blandos; aunque nuestro paciente no presentaba estas características, siempre se deben considerar como factores predisponentes.

La alta prevalencia, asociada con la creciente evidencia de infecciones asociadas, aporta relevancia para la comprensión de los mecanismos fisiopatológicos de la enfermedad. De manera similar al lipopolisacárido gramnegativo, que tiene la capacidad de aumentar la susceptibilidad al choque, se ha demostrado previamente la producción de exotoxina pirogénica en estreptococos ${ }^{6}$.

Estos superantígenos evaden la secuencia habitual de respuesta inmunitaria mediada por antígenos y activa el sistema inmunitario, lo que resulta en la liberación de grandes cantidades de citocinas inflamatorias y el consiguiente fallo multiorgánico. Los estudios han demostrado que los sueros de cultivo concentrados que contienen SGB no reaccionan con antisueros contra las exotoxinas pirogénicas $A, B$ y $C$ de los estreptococos, que son exotoxinas bien documentadas de estreptococos del grupo A, lo que sugiere la existencia de una o más toxinas pirogénicas de SGB no caracterizadas ${ }^{7}$. Son necesarios estudios para caracterizar completamente estos antígenos.

Hasta donde sabemos, en la literatura hay 32 casos documentados de síndrome de shock tóxico por S. agalactiae ${ }^{7}$. Los pacientes de edad avanzada son particularmente propensos al desarrollo de enfermedades graves. La comorbilidad estuvo presente en el $37.5 \%$ de los casos $^{8}$. Condiciones médicas como malignidad, cirrosis y diabetes mellitus se asocian con un mayor riesgo ${ }^{8}$.

El tratamiento del shock tóxico por estreptococos requiere un enfoque multidisciplinario, con medidas de apoyo inmediatas, un régimen antibiótico apropiado y, finalmente, intervención quirúrgica en algunos casos. La penicilina es el antibiótico de elección para la infección por SGB, pero la sensibilidad suele extenderse a la ampicilina, las penicilinas de espectro extendido y las cefalosporinas.
La resistencia a las fluoroquinolonas, aunque poco común en Occidente, es una preocupación creciente en China, Corea del Sur y Japón, donde el número de cepas resistentes al ciprofloxacino alcanza el $42.1 \%$.

Debido a que el SGB se ha abordado recientemente como una causa importante de infecciones invasivas en los adultos mayores y en pacientes con trastornos subyacentes, estos sujetos podrían representar poblaciones objetivo para las medidas preventivas ${ }^{10}$.

La inmunogenicidad de los polisacáridos como vacunas humanas se mejora mediante la conjugación con portadores de proteínas, lo que induce una respuesta inmunitaria robusta y duradera contra el polisacárido. Las vacunas monovalentes conjugadas que incluyen los serotipos causantes de enfermedades más frecuentes, junto con el toxoide tetánico, se probaron en ensayos clínicos de fase I y fase II en mujeres sanas y demostraron mejorar la inmunogenicidad, que dependía de la dosis y era más consistente con una respuesta de memoria ${ }^{10}$.

Solo un estudio clínico se centró en adultos sanos mayores de 65 años y la respuesta inmunitaria tras la administración de una vacuna conjugada de polisacáridos capsulares y toxoide tetánico de tipo $\mathrm{V}$ no mostró diferencias estadísticamente significativas en la respuesta inmunitaria en comparación con adultos de 18 a 50 años $^{11}$. Son necesarios estudios adicionales para comprender si el mismo efecto podría obtenerse en personas mayores con afecciones médicas subyacentes.

\section{Conclusiones}

- El reconocimiento rápido del síndrome de shock tóxico exige una alta sospecha clínica y el diagnóstico debe considerarse en todo paciente con inicio brusco de choque sin una etiología clara, principalmente con coexistencia de lesiones cutáneas.

- El uso de antibióticos que inhiben la síntesis de proteínas bacterianas es crucial para reducir la gravedad de la enfermedad. Se debe preferir el linezolid en áreas de alta resistencia a la clindamicina.

- Se necesitan más estudios para comprender mejor la respuesta inmunitaria de los mecanismos fisiopatológicos, permitiendo una mejor profilaxis de la población en riesgo y el entendimiento de la medicina más personalizada según cada paciente y sus factores de riesgo.

- El papel de la vacunación parece prometedor, por lo cual es importante considerarlo en todos los pacientes mayores de 65 años, en especial en aquellos con factores de riesgo. 


\section{Financiamiento}

Los autores declaran no haber recibido financiamiento para este estudio.

\section{Conflicto de intereses}

Los autores declaran no tener ningún conflicto de intereses.

\section{Responsabilidades éticas}

Protección de personas y animales. Los autores declaran que para esta investigación no se han realizado experimentos en seres humanos ni en animales.

Confidencialidad de los datos. Los autores declaran que han seguido los protocolos de su centro de trabajo sobre la publicación de datos de pacientes.

Derecho a la privacidad y consentimiento informado. Los autores han obtenido el consentimiento informado de los pacientes y/o sujetos referidos en el artículo. Este documento obra en poder del autor de correspondencia.

\section{Bibliografía}

1. Lamagni TL, Keshishian C, Efstratiou A, Guy R, Henderson KL, Broughton $\mathrm{K}$, et al. Emerging trends in the epidemiology of invasive group $\mathrm{B}$ streptococcal disease in England and Wales, 1991-2010. Clin Infect Dis. 2013;57:682-8.

2. Gallagher PG, Watanakunakorn C. Group B streptococcal bacteremia in a community teaching hospital. Am J Med. 1985;78:795-800.

3. Verghese A, Mireault K, Arbeit RD. Group B streptococcal bacteremia in men. Rev Infect Dis. 1986;8:912-7.

4. Opal SM, Cross A, Palmer M, Almazan R. Group B streptococcal sepsis in adults and infants: contrasts and comparisons. Arch Intern Med. 1988; 148:641-5.

5. Lerner PI, Gopalakrishna KV, Wolinsky E, Mchenry MC, Tan JS, Rosenthal M, et al. Group B streptococcus (S. agalactiae) bacteremia in adults: analysis of 32 cases and review of the literature. Medicine. 1977;56:457-73.

6. Al Akhrass F, Abdallah L, Berger S, Hanna R, Reynolds N, Thompson S, et al. Streptococcus agalactiae toxic shock-like syndrome: two case reports and review of the literature. Medicine. 2013;92:10-4.

7. Meireles M, Ricardo M, Ribeiro S, Araújo Correia J. Streptococcus agalactiae toxic shock syndrome: new agent, new challenges. A case report and literature review. Infect Dis Clin Pract. 2017;25:66-70.

8. Jackson LA, Hilsdon R, Farley MM, Harrison LH, Reingold AL, Plikaytis BD, et al. Risk factors for group B streptococcal disease in adults. Ann Intern Med. 1995;123:415-20.

9. Crum NF, Wallace MR. Group B streptococcal necrotizing fasciitis and toxic shock-like syndrome: a case report and review of the literature. Scand J Infect Dis. 2003;35:878-81.

10. Nuccitelli A, Rinaudo CD, Maione D. Group B Streptococcus vaccine: state of the art. Ther Adv Vaccines. 2015;3:76-90.

11. Palazzi DL, Rench MA, Edwards MS, et al. Use of type V group B streptococcal conjugate vaccine in adults $65-85$ years old. $J$ Infect Dis. 2004;190:558-564. 